\title{
Intellectual modeling of surface heat-exchange enhancer based on artificial neural networks
}

\author{
K. K. Gilfanov ${ }^{1}, R$. A. Shakirov ${ }^{1, *}$ \\ ${ }^{1}$ Kazan State Power Engineering University, Kazan, Russia
}

\begin{abstract}
The results of neural network modeling of average heat transfer in the channels of exchangers with surface enhancer of different shapes are presented. Artificial neural networks are trained using experimental data, which covers more than ten sources. The possibility and prospects of building artificial neural networks for modeling the characteristics of heat exchange surfaces are shown.
\end{abstract}

\section{Introduction}

Currently, all the generated energy before its use is repeatedly converted with various heat exchangers; therefore, the efficiency of the entire production depends on their efficiency. Increasing the efficiency of modern heat exchangers is associated with the use of passive methods of heat transfer enhancement. Positive practical effect showed surface enhancer in the form of collar steps and spherical protrusions. The physical principles of this method of enhancement have been known for a long time. However, quantitative dependences for heat transfer and frictional head coefficients calculation in a broad turndown of geometrical parameters and performance characteristics, are not fully defined yet. This method of heat transfer enhancement is characterized by a technological effectiveness and high thermohydraulic efficiency [1].

There is a huge heat transfer enhancement database in the technical literature, which is estimated at more than 9000 technical articles, papers and reports, and published in periodicals and numerous bibliographical reports of A. E. Bergles, M. K. Jensen and B. Some, in reviews by R. Webb, D. P. Shatto and John. P. Peterson, A. E. Bergles, R. M. Manglik, in monographs of R. Thomas, R. Webb, R. M. Manglik and A. D. Kraus, S. Kakuta, K. X. Pressure, and etc. [2].

Passive methods of heat transfer enhancement reduce the thermal resistance of wall layers in convective heat transfer near the heat exchange surface, contributing to an increase in the film conductance coefficient. As a result, there is a possibility of heat exchangers' dimensions and weights reduction without capacitance rate changing or a significant increase in the capacitance rate at the current dimensions and weights of heat exchangers.

Calculation and design of the intensified heat exchangers with optimal characteristics is complicated by research results generalization problem. The analysis of literature sources shows that it is impossible to generalize the characteristics of surface heat transfer enhancements by conventional conservation equations due to the complexity of thermal and hydro-mechanical processes. If it is possible to generalize the results of the research in the form of empirical formulas of the dependence of the Nusselt numbers on the Reynolds and Prandtl numbers with the involvement of the determining dimensions, then, as a rule, for a narrow class of single recesses or systems of recesses in the range of parameters of the experiments [3].

A certain way out of the situation is offered by artificial intelligence systems capable of learning or selflearning. These are artificial neural networks (ANN). In the ANN, data is kept as multiple neurons and the connections between them. A neural network with a certain number of inputs and outputs is constructed from neurons (nodes) connected in a certain way. Neural network modeling allows to generalize the results of the experiments of complex multiparameter processes, "looking" beyond the range of parameters in which the experiments were conducted. In neural networks, knowledge is contained in the states of many neurons and connections between them. The neural network model is a trained computer program that is able to predict the optimal parameters of the heat exchange surface.

\section{Neural network modeling}

Artificial neural network is implemented using a neural network development environment "NeuroSolutions". It contains a master of neural network architectures ("NeuralWizard"), which is used to set the architecture, select the training sample, and training criteria. When solving the task, we used a version of the program with full functionality.

The training set vectors are presented sequentially, errors are calculated, and weights are adjusted for each vector until the error across the training array reaches an acceptably low level. To set the initial values of the

\footnotetext{
*Corresponding author: Shakirov.aiv@yandex.ru
} 
weight coefficients, a statistical setting is used, designed to slightly improve the initial initialization algorithm on the basis of additional information on the data.

The principles of optimality are formulated as follows: for each input neuron, the change of input variables in the range of true levels should have approximately the same change in the output signal of the neuron and the level of displacement for each neuron should be set so as to provide an average maximum value of the derivative for the activation function of the training sample (Taskbook).

To develop an artificial neural network and its training, the experimental material on heat exchange of surfaces with collar steps in the form of dependence of relative Nusselt numbers $\mathrm{Nu}$ on Reynolds numbers Re was used [1-4].

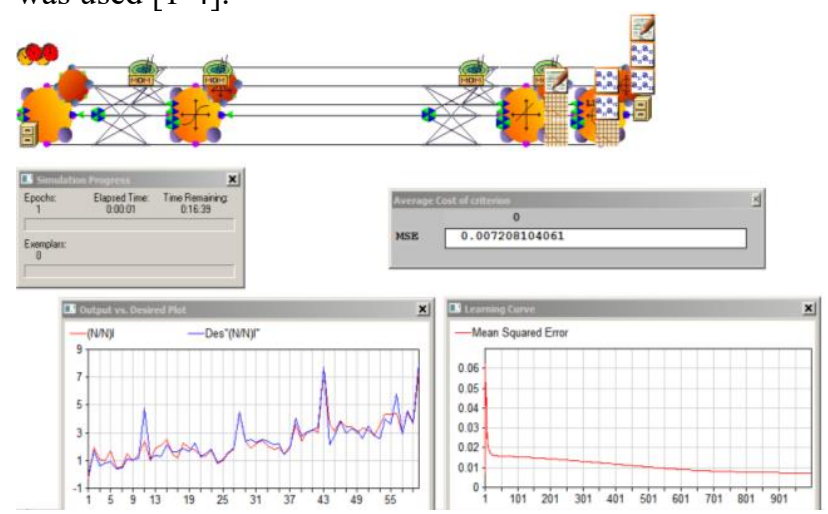

Fig. 1. Structure and training of an ANN.

In accordance with the experimental data of studies, the parameters of the artificial neural network are adjusted, its structure is created and the network is trained. The structure and process of is training are shown in Figure 1.

After training an artificial neural network, it is necessary to test it. To test our trained ANN, we use a matrix with 'unknown data', which are also selected from [1-4], but are not included in the 'teacher' array. We need to model (predict) the output of the quantity $(\mathrm{Nu} / \mathrm{Nu} 0) \mathrm{Re}$. The test results for each type of enhancers are presented below.

\subsection{Collar steps}

Collar steps have proven to be high thermal efficiency and manufacturability. Despite the rather large number of works on this subject, in the modern world literature there is a limited number of works devoted to the study of heat transfer enhancement in laminar and transient flow regimes on surfaces with transverse collar steps [5].

The results of testing a trained artificial neural network on the basis of experimental research data for surfaces with collar steps are presented in Figure 2, where (Des) are the actual values, and (Out) are the values predicted by the developed network.

The mean square error of neural network approximation, which is given by our program, is about $1.146 \%$.

\subsection{Spherical protrusions}

Compared with the collar steps, spherical protrusions have an advantage, because they narrow the flow section of the pipe significantly less. In the scientific and technical literature there is a limited number of publications devoted to the study of the thermohydraulic characteristics of the paths with spherical projections [6].

Figure 3 presents the of artificial neural network testing results for experimental data, which presents the real values (Des) and the values predicted by our network (Out).

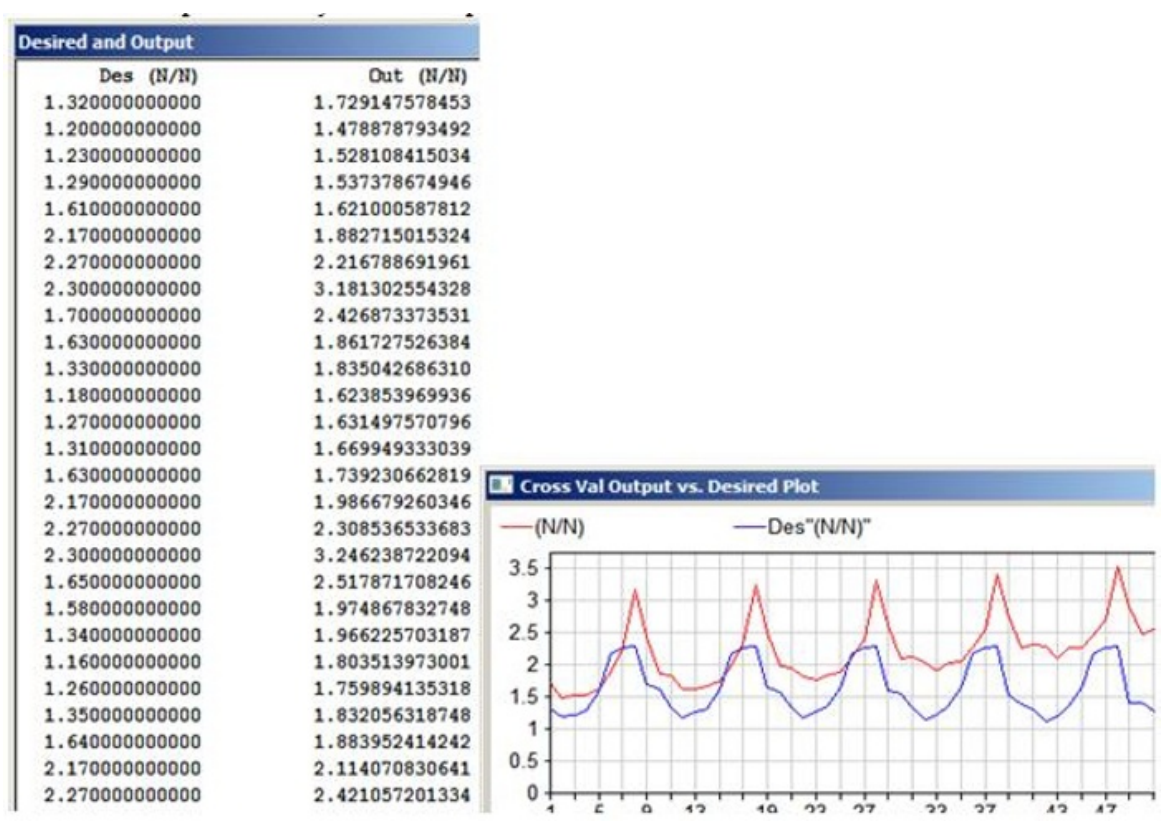

Fig. 2. Screenshots of the actual values of the experimental matrix and network testing results for collar steps. 


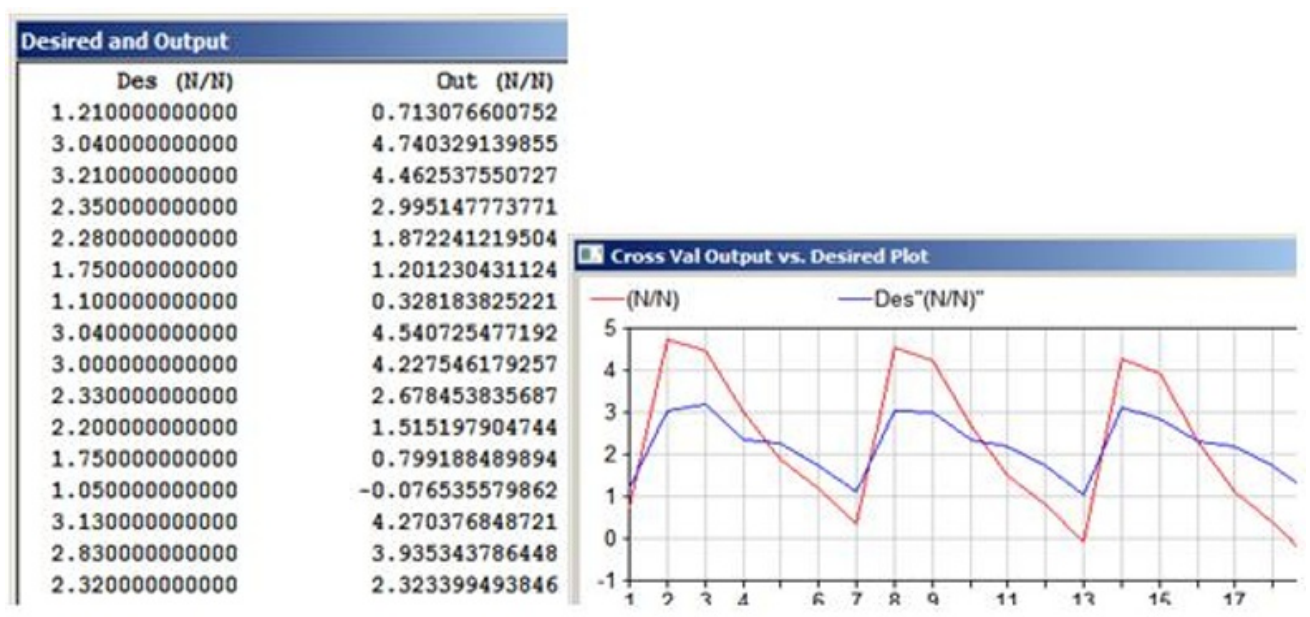

Fig. 3. Screenshots of the actual values of the experimental matrix and network testing results for spherical protrusions.

The standard of neural network approximation, which is given by our program, is about $1.782 \%$.

\subsection{Spherical recesses}

It is established that a non-separable spherical profiling of the recesses with rounded edges allows you to implement a confuser-diffuser flow. There are no any large-scale vortex structures in the recess. It is this flow regime that provides an outstripping increase in heat transfer over the growth of frictional head [7].

Intermediate results of neural network modeling of spherical recesses, where the possibility of effective neural network modeling of surface heat transfer

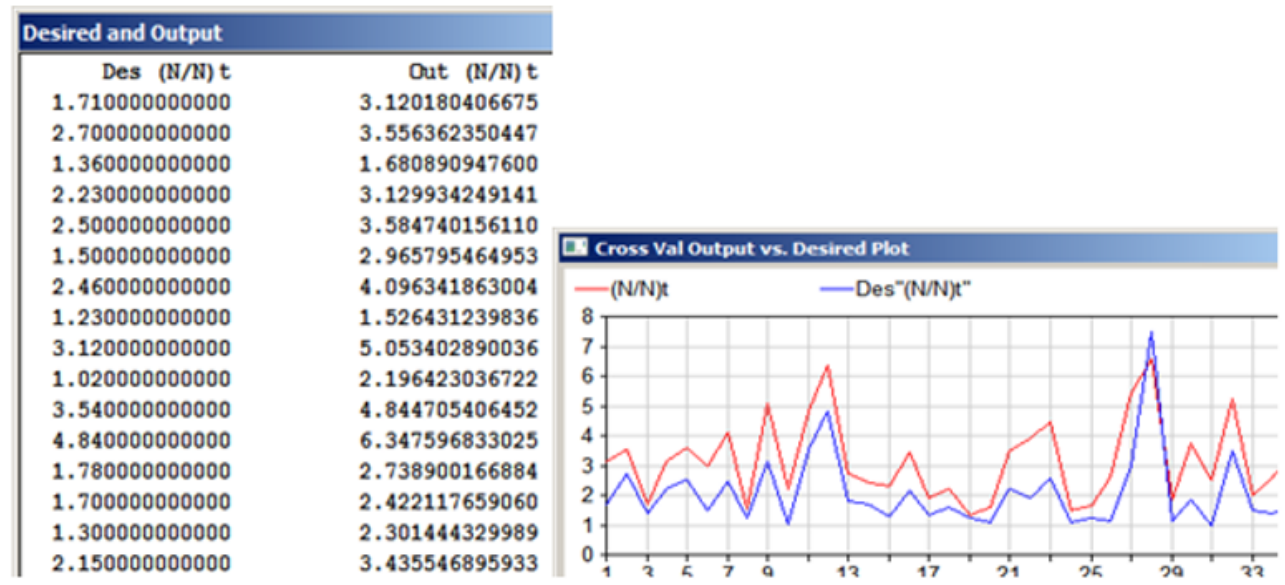

Fig. 4. Screenshots of the real values of the experimental matrix and the results of testing the network for the turbulent regime of channels with spherical recesses.

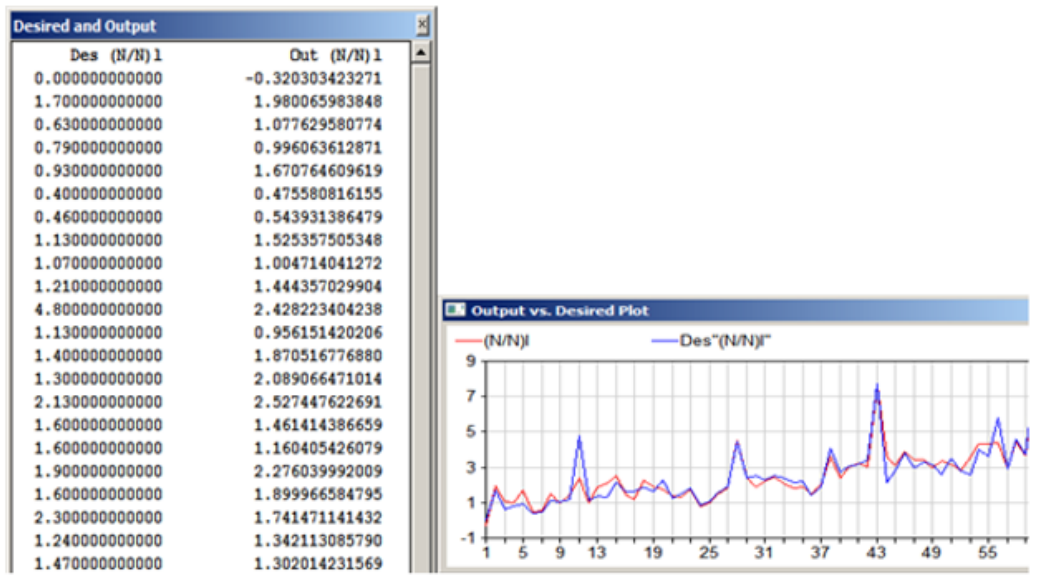

Fig. 5. Screenshots of the real values of the experimental matrix and the results of network testing for the laminar regime of channels with spherical recesses. 
enhancers was shown, are presented hereinbefore. In continuation of this work, on the basis of experimental data of the study [8] artificial neural networks for laminar and turbulent flow were developed and trained.

The test results of the developed artificial neural network are presented in Figures 4 and 5, where (Des) are the actual values and (Out) are the values predicted by our network.

The standard error of neural network approximation, which is given by the program, is about $0.72 \%$. Also in this neural network model, the results of the following studies are used [9-16].

\section{Conclusions}

Thus, the possibility of constructing artificial neural networks for modeling surface heat transfer enhancers in the form of collar steps, spherical recesses and protrusions is presented. Testing of the neural network showed a modeling error from $0.7 \%$ to $1.7 \%$, which can be considered satisfactory, taking into account the spread in the sample data associated with the error of the basic experimental data (5-15) \%. It should also be noted that for the practical use of the simulation results it is necessary to expand the range of data on surface heat transfer enhancers. The neural network model can be used to optimize heat exchange surfaces under different operating conditions.

\section{References}

[1] A.V. Shchelchkov Int. Conf. on Vortex Flows and Vortex Models (Rostock: ICVFM) 11, (2016)

[2] Y.F. Gortyshov, I.A. Popov, A.B. Shchelchkov, V.V. Olimpiev, S.I. Kaskov, Thermohydraulic efficiency of the perspective ways of intensification of heat transfer in channels heat transfer equipment (Kazan: Center of innovative technologies) (2009)

[3] S.A. Isaev, Numerical simulation of the intensification of the heat exchange in a planeparallel channel with a cylindrical shallow dimple on the heated engineering Physics and Thermophysics 5(89), 11861201 (2016)

[4] E.K. Kalinin, G.A. Dreitzer, I.Z. Kopp, A.S. Macachin, Effective heat transfer surfaces (Moscow: Energoatomizdat) 101-102 (1998)

[5] Yu.I. Shanin, O.I. Shanin, Intensification of heat transfer by application of spherical holes on the channel walls Convective heat and mass transfer (Minsk: ANB) (2004)

[6] I. Borisov, A. Khalatov, S. Kobzar, B. Glezer, Comparison of thermal hydraulic characteristics for two types of dimpled surfaces ASME GT2004-54204 (2004)

[7] Yu.F Gortyshov., I.A. Popov, A.B. Clicks, D.V. Ryzhkov, Heat-Hydraulic characteristics of heat exchangers with surface intensification of heat exchange in the form of spherical recesses and protrusions (Moscow: MEI) (2008)
[8] K.Kh. Gilfanov, R.A. Shakirov, R.N. Gainullin Bulletin of technological university 21, 102105; (2019)

[9] S.A. Isaev, A.V. Schelchkov, A.I. Leontiev, P.A. Baranov, M.E. Gulcova Int. J. Heat and Mass Transfer 94, 426-448 (2016)

[10] Y. Rao1, Y. Feng, B. Li, B. Weigand J. Heat Transfer 031901-10, 137 (2015)

[11] S.C. Lau, S.W. Moon, Journal of Energy, Heat and Mass Transfer 23, 393-416 (2001)

[12] W. Chakroun, M.M.A. Quadri, Int. J. Therm. Sci. 2(41), 163-172 (2002)

[13] A.D. Ochoa, J.W. Baughn, A.R. Byerley, Int. J. of Heat and Fluid Flow. 26, 264-275 (2005)

[14] O. Marsden, C. Bogey, C. Bailly, 16th AIAA/CEAS Aeroacoustics Conf. (Stockholm: AIAA) $1-13(2010)$

[15] J. Turnow, Flow structure and heat transfer on dimpled surfaces PhD Thesis University of Rostock 151 (2011)

[16] S.A. Isaev, A.I. Leontiev, A.V. Schelchkov, M.E. Gul'tcova, Journal of Engineering Physics and Thermophysics 5(88), 1304-1308 (2015) 